\title{
An OFDMA-Based Next-Generation Wireless Downlink System Design with Hybrid Multiple Access and Frequency Grouping Techniques
}

\author{
Won-Ick Lee, Byeong Gi Lee, Kwang Bok Lee, and Saewoong Bahk
}

\begin{abstract}
This paper discusses how to effectively design a nextgeneration wireless communication system that can possibly provide very high data-rate transmissions and versatile quality services. In order to accommodate the sophisticated user requirements and diversified user environments of the next-generation systems, it should be designed to take an efficient and flexible structure for multiple access and resource allocation. In addition, the design should be optimized for cost-effective usage of resources and for efficient operation in a multi-cell environment. As orthogonal frequency division multiple access (OFDMA) has turned out in recent researches to be one of the most promising multiple access techniques that can possibly meet all those requirements through efficient radio spectrum utilization, we take OFDMA as the basic framework in the next-generation wireless communications system design.

So, in this paper, we focus on introducing an OFDMA-based downlink system design that employs the techniques of hybrid multiple access (HMA) and frequency group (FG) in conjunction with intra-frequency group averaging (IFGA). The HMA technique combines various multiple access schemes on the basis of OFDMA system, adopting the multiple access scheme that best fits to the given user condition in terms of mobility, service, and environment. The FG concept and IFGA technique help to reduce the feedback overhead of OFDMA system and the other-cell interference (OCI) problem by grouping the sub-carriers based on coherence bandwidths and by harmonizing the channel condition and OCI of the grouped sub-carriers.
\end{abstract}

Index Terms: Downlink, multi-cell environment, multiple access, OFDMA.

\section{INTRODUCTION}

The next-generation wireless communication systems beyond the 3rd generation are expected to provide very high data-rate transmissions and versatile quality services. According to ITU reports, the next-generation wireless communications systems are required to provide a $100 \mathrm{Mbps}$ data rate for fast moving users and a 1 Gbps data rate for fixed users, which is $50 \sim 100$ times higher than that of $3 \mathrm{G}$ systems [1].

In order to be able to provide such high data rate services, it is necessary to employ advanced multiple access and transmission technologies that can utilize frequency resources most efficiently. Among various multiple access techniques known to date, orthogonal frequency division multiple access (OFDMA)

Manuscript received February 15, 2005.

The authors are with the School of Electrical Engineering, Seoul National University, Seoul, 151-742, Korea, email: 1wi@tsp.snu.ac.kr, \{blee, klee, sbahk\}@snu.ac.kr.

This work was supported in part by Samsung Advanced Institute of Technology. is one of the most favored schemes for use in broadband transmission system design as it is capable of overcoming intersymbol interference (ISI) and utilizing the given radio spectrum efficiently by dividing it into a large number of small subbands [2], [3]. There have been reported a number of OFDMAbased system designs, such as adaptive OFDMA [2], frequency hopping (FH) OFDMA [4], [5], and multi-carrier code division multiple access (MC-CDMA) [6]-[8] systems. In addition, there are various other techniques that have been introduced to help enhance the spectral efficiency through resource management algorithms such as opportunistic scheduling [9]-[13] and adaptive channel allocation [14]-[19]. Such link adaptation techniques are tightly coupled with the physical layer structures and feedback mechanisms, so in the relevant system design, transmission technologies should be considered in combination with resource management schemes.

In addition to the requirements on high data rate transmission and versatile quality services, there are various other requirements that should be incorporated in designing the nextgeneration wireless communications systems: Basically, the system should be designed to efficiently provide services to users in different conditions in terms of mobility, service requirements, and geographical environment. Secondly, it should be optimized to effectively utilize the limited frequency spectrum in multi-cell environment. Third, the cell planning should be done to be effective for distributed cellular systems with universal frequency reuse. Fourth, the system should be capable of overcoming other-cell interference (OCI) caused by universal frequency reuse and be required to minimize the level of coordination among the neighboring cells. Fifth, the architecture should be designed to render an easy connection and/or expansion to IP networks.

In order to accommodate such sophisticated requirements, we need to design the next-generation wireless communication systems to be efficient and flexible in handling multiple access, resource allocation and management and to be robust to OCI. In this paper, we introduce a next-generation system design, focusing on its downlink part. The system design satisfies all these requirements by taking OFDMA technology as the basic framework while employing different variations for different user conditions. The resulting system is composed of three major building blocks: The hybrid multiple access (HMA) technique, the frequency group $(\mathrm{FG})$ technique, and the intra frequency group averaging (IFGA) technique. A major portion of the paper will be dedicated to discussing the reasons why HMA, FG, and IFGA techniques are conceived and how these techniques are to be implemented. 
The HMA technique is designed to combine various multiple access mechanisms on the basis of OFDMA system and choose the most appropriate multiple access technique depending on the mobility, service, and environment of the user. The OFDMA framework that adopts the HMA technique renders a solid basic structure to build an efficient and flexible downlink system in which a diverse set of access techniques and resource management algorithms can be effectively combined to provide the desired user services and QoS requirements.

The FG technique, in conjunction with the IFGA technique, is designed to reduce the feedback and control overheads, since the OFDMA system that exploits multi-user diversity gain through resource management inherently causes heavy control overhead. We form each FG by grouping the sub-carriers within a coherence bandwidth so that the signal-to-noise-ratio (SNR) may be regarded identical among all the sub-carriers belonging to the same FG. Then the feedback and pilot overheads reduce significantly as feedback can be done FG based, not sub-carrier based. In addition, we adopt the IFGA technique such that signal-tointerference and noise-ratio (SINR) is maintained uniform over all sub-channels within the same FG by averaging out the channel characteristics of each constituent sub-channel. Such IFGA technique makes the FG concept effectively applicable to multicell OFDMA systems, by overcoming the OCI variation problem caused by non-uniform resource usage in the neighboring cells.

This paper is organized as follows. We first develop the HMA technique in Section II and present the frame structure that employs the FG concept in Section III. Then in Section IV, we discuss how to improve the structure by adopting the IFGA processing and analyzing the performance of different types of IFGA mechanisms. Finally, we draw conclusions on the HMA based downlink system design in Section V.

\section{HYBRID MULTIPLE ACCESS (HMA)}

As discussed above, the next-generation wireless communication systems should be designed to be capable of accommodating sophisticated user requirements and diversified user environments. So they should be designed to take an efficient and flexible structure for multiple access and resource allocation such that they become optimized for cost-effective utilization of resources and efficient operation in multi-cell environment. However, the existing OFDMA-based multiple access schemes retain both advantages and disadvantages that vary depending on the application requirements and the user conditions [3]. Specifically, OFDMA itself can achieve high spectral efficiency but is not adequate for use in mobile or high OCI environment; FHOFDMA can provide frequency diversity gain with light control overhead so it is adequate for mobile users but it sacrifices the spectral efficiency; and MC-CDMA can provide OCI robustness and frequency diversity gain but suffers from inter-code interference and channel estimation problems. ${ }^{1}$ Therefore, in the next-generation system design it is desirable not to single out one access technique among them to cover all different users,

\footnotetext{
${ }^{1}$ In the MC-CDMA system, accurate channel estimation for each sub-carrier is required to despread the frequency-spread signals without inter-code interference, which requires high pilot and system overhead.
}

each-with different QoS requirements, mobility and environments, but to mix and match different access techniques depending on the application requirements and user conditions.

In order to take advantage of the different features possessed by different multiple access techniques, we take a top-down approach that adopts the concept of class to categorize users depending on the QoS requirement, mobility and environment. Based on this categorization, we build a hybrid multiple access scheme that chooses the most appropriate multiple access technique for each different class, thereby maximizing the efficiency and flexibility in multiple access.

\section{A. Mobility Classes}

In the aspect of user mobility, we may classify users into two mobility classes depending on the speed of user's movementmobile users and nomadic users.

In the case of the nomadic users, the channel conditions change slowly as the Doppler frequency spread due to the movement speed is small. This helps the nomadic users utilize link adaptation with little feedback overhead. So in designing the downlink system for nomadic users we may set the main objective to fully utilize the radio spectrum and achieve high spectral efficiency based on adaptive resource management such as adaptive modulation and coding (AMC) and wireless scheduling algorithms over adaptive OFDMA. We may exploit multiuser diversity by selecting the most appropriate sub-carrier for each user and applying AMC according to long-term and shortterm channel qualities, respectively, or by applying opportunistic scheduling algorithms over the shared channels. However, it is not advantageous to apply adaptive resource management to nomadic users in unfavorable environments or with simple service requirements. Therefore, we need to determine the most appropriate multiple access technique and resource allocation strategy after considering the service requirement and user environment. So we defer further discussions on detailed selection schemes for nomadic users to the next subsections.

On the other hand, in the case of mobile users, the channel conditions change fast due to the high Doppler frequency spread caused by high mobile speed. Then the link adaptation techniques become meaningless as the feedback information may be hardly delivered to the controller within some meaningful time duration, or the coherence time. Consequently, we need to set the main design objective of the downlink system to provide robust services under fast-varying channel conditions. In this case, frequency diversity gain and control overhead become more important factors than the spectral efficiency. Therefore, it is desirable, under the HMA framework, to take FH-OFDMA as the opted multiple access scheme for mobile users. Differently from the case of nomadic users, we may not consider the effects of the service and environment classes for the mobile users. The reason is that the application of link adaptation techniques ${ }^{2}$ does not enhance the spectral efficiency for mobile users due to the short coherence time.

It is possible to consider MC-CDMA as another candidate multiple access technique for mobile users. However, MCCDMA requires accurate channel estimation per each sub-

\footnotetext{
${ }^{2}$ In the HMA framework, service and environment classes are considered for further enhancement of the spectral efficiency through link adaptation.
} 


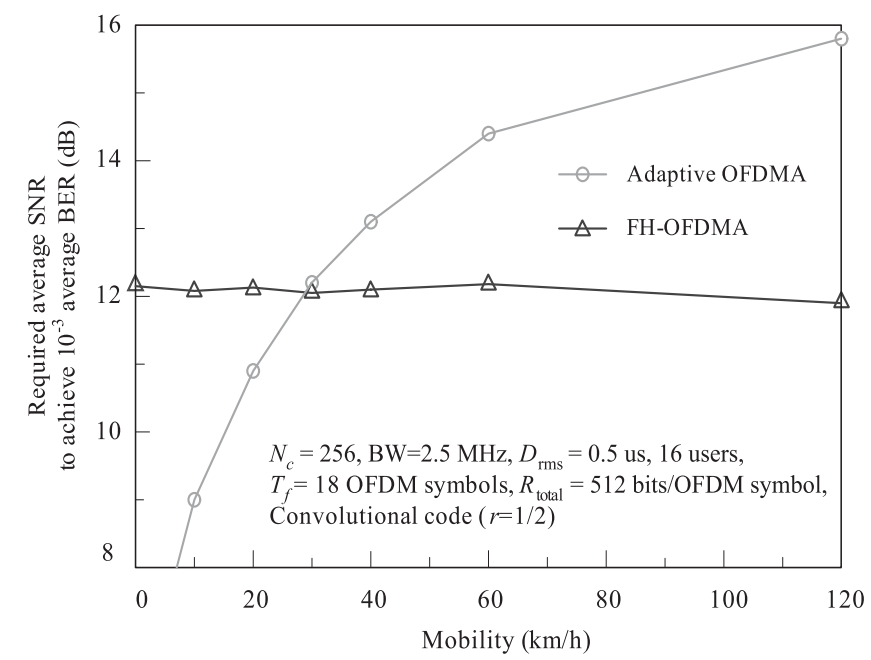

Fig. 1. Performance comparison of adaptive OFDMA and FH-OFDMA with respect to mobility.

carrier for despreading but the channel estimation errors and the corresponding inter-code interference are likely to increase as the mobile speed increases. Therefore, under the HMA framework, we take FH-OFDMA as the opted multiple access mechanism for mobile users.

Fig. 1 shows the simulated performances of FH-OFDMA and adaptive OFDMA ${ }^{3}$ techniques with respect to various mobility speeds. The simulation was performed over an OFDM system with 256 sub-carriers in a $2.5 \mathrm{MHz}$ bandwidth and a 115.2 $\mu$ s symbol length (including cyclic prefix length of $12.8 \mu \mathrm{s}$ ). We assumed that the frame length is 18 OFDM symbols (i.e., $2.074 \mathrm{~ms}$ ) and the link adaptation is performed for the adaptive OFDMA at every frame and with one-frame feedback delay. We assumed perfect channel estimation and no feedback error. For the channel model, we used a 5-path Rayleigh fading channel with wide-sense stationary uncorrelated scattering. In time domain, we used Jakes model and exponential decaying delay profile model with $D_{\mathrm{rms}}=0.5 \mu \mathrm{s}$ and set the path loss exponent to 3.5. The delay distribution of each Rayleigh path is generated by a uniform distribution with the mean $D_{\mathrm{rms}}$. Plotted in the figure is the SNR required to achieve a total rate of 512 bits/OFDM symbol with a $10^{-3}$ BER constraint using AMC. We used QPSK, 16-QAM, and 64-QAM for modulation schemes and rate $1 / 2$ convolutional code with length 7 for channel coding. During the simulation, we applied equal power allocation and adapted the modulation set for each sub-carrier through the adaptive sub-carrier allocation (ASA) and adaptive bit allocation (ABA) algorithms [17] in order to minimize the BER with the constraint of the total rate. We do not adapt the code rate in order to reduce the simulation complexity.

The SNR requirement value in the figure is the average of the performances of 16 users. From the figure, we observe that the FH-OFDMA maintains good performance even when the mobile speed increases but the adaptive OFDMA exhibits good performance only when the mobile speed is less than $30 \mathrm{~km} / \mathrm{h}$. This result testifies that the FH-OFDMA is an adequate choice

\footnotetext{
${ }^{3}$ Adaptive OFDMA refers the OFDMA system that adopts dynamic channel allocation algorithms.
}

for mobile users.

In the HMA framework, we may differentiate nomadic and mobile users depending on the utilization of link adaptation. A user $i$ estimates its own channel coherence time $C C T_{i}$ through the pilot signal received from the base station. If the estimated $C C T_{i}$ value is longer than the minimum threshold value required for link adaptation, $T_{L A}$, we may classify the user as nomadic and, otherwise, as mobile. For determining the threshold value $T_{L A}$, we should consider the unit time needed for link adaptation and the feedback delay. We will defer the discussion of this issue to Section III-B.

\section{B. Service Classes}

In the aspect of services, we may classify users based on the two well-known classes-real-time (RT) services and non realtime (NRT) services.

As the NRT service refers to bursty data service such as web browsing, FTP and emails, throughput is the main metric of QoS and delay is not of much concern. So the multi-user diversity gain for NRT services may be maximally exploited by applying an opportunistic scheduling algorithm over the shared channel in such a way that the user in the best condition of channel quality and service statistics is selected for service. The scheduling algorithm may be selected among the multitude of wireless scheduling algorithms that have been reported in the literature to date [9]-[13].

In order to fully exploit such multi-user diversity, we assign shared-OFDMA (S-OFDMA) channel to the nomadic users that require NRT services. As there exist multiple S-OFDMA channels and multiple users, it is necessary to group users to share the same S-OFDMA channel by considering the service requirements and environment. In the proposed HMA framework, we dynamically assign up to eight users into an S-OFDMA channel and adopt the CDF-based scheduling (CS) algorithm [12] for opportunistic scheduling. When a new user requests an NRT service, we select an appropriate existing S-OFDMA channel or a new S-OFDMA channel depending whether or not the assignment of the new user violates the QoS requirement of the readily admitted users. Such an assignment scheme makes sense, as it is possible to estimate the throughput performance of each user at the call admission control stage by adopting the CS algorithm [12]. On the other hand, if the NRT service is such a one that requires a huge bulk of data transmission, such as FTP service, we can assign a dedicated channel to shorten the data transmission time.

In the case of RT services such as voice and streaming services, it is hard to apply the opportunistic scheduling algorithms, as RT services produce delay-sensitive traffic. So dedicated channels, such as the dedicated-OFDMA (D-OFDMA) channel or the FH-OFDMA channel, are more appropriate to assign than the shared channels such as the S-OFDMA channel. If a higher spectral efficiency is desired, it is possible to apply adaptive channel allocation algorithms [14]-[17] to D-OFDMA channel, instead of the opportunistic scheduling algorithms. However, if the required RT service is a low data-rate service such as a typical voice service, FH-OFDMA is the better choice, as the control and feedback overheads required to apply the adaptive channel allocation algorithm would be larger than the gain ob- 
tained by applying it. In the HMA framework, we arrange a D-OFDMA channel to consist of $S C_{L A}$ or more downlink subcarriers of identical channel condition ${ }^{4}$ and a feedback channel in order to reduce the feedback and control overheads. This means that if an RT service uses a D-OFDMA channel, it should use at least $S C_{L A}$ sub-carriers. So, unless the number of FHOFDMA channels, $N_{\mathrm{FH}}$, required to serve an RT service does not exceed $S C_{L A}$, it is more efficient to use $N_{\text {FH }}$ FH-OFDMA channels than to use one D-OFDMA channel. Note that one FH-OFDMA channel is equivalent to one sub-carrier of the DOFDMA channel. Therefore, under the HMA framework, a nomadic user of RT services first estimates the $N_{\mathrm{FH}}$ value and then selects an appropriate multiple access scheme based on this value. The condition to select FH-OFDMA is

$$
N_{\mathrm{FH}} \leq S C_{L A}
$$

\section{Environment Classes}

In the cellular system, the environment of a user highly depends on its location and the resource utilization of the neighboring cells. In this aspect, we may consider classifying the geographical user environment into two classes depending on the proximity to the center of a cell-cell center and cell boundary.

If a user were located at the cell boundary, it would experience not only the low average SNR but also high OCI, with the OCI fluctuation affecting the SINR more than the SNR fluctuation does. This fact implies that if a user approaches the cell boundary, the multi-user diversity gain obtained by adopting adaptive D-OFDMA diminishes as the average SINR needed to select the appropriate D-OFDMA channel varies when the resource allocation of neighboring cells changes. On the contrary, the frequency diversity gain of FH-OFDMA becomes more effective since not only the instantaneous SINR but also the average SINR of the OFDMA sub-carriers fluctuates.

Fig. 2 shows the simulated performances of FH-OFDMA and adaptive OFDMA applied to differently located users with respect to different values of interference loading factor (ILF), which is defined by

$$
\mathrm{ILF}=\frac{\text { Number of used subcarriers in neighboring cells }}{\text { Number of total subcarriers in neighboring cells }} .
$$

In the simulation, we took the typical 7-cell structure and allocated sub-carriers in the neighboring cells such that the ILF value becomes identical for each neighboring cell. Specifically, we assigned both D-OFDMA channels and FH-OFDMA channels in each neighboring cell to generate a semi-dynamic OCI model. For each neighboring cell, we allocated $1 / 2 \delta N_{T}$ subcarriers to D-OFDMA channels in a random fashion and assigned 1/2 $\delta N_{T}$ FH-OFDMA channels that hop randomly each symbol period among the other $(1-1 / 2 \delta) N_{T}$ sub-carriers, for the ILF value $\delta$ and the total number of sub-carriers in each cell $N_{T}$. Under such OCI model, some sub-carriers would experience constant OCI due to the D-OFDMA channels allocated in the neighboring cells and some others would experience dynamically changing OCI due to the FH-OFDMA channels assigned

\footnotetext{
${ }^{4}$ We can provide multiple sub-carriers of identical channel condition by adopting the FG concept and IFGA processing, as will be described in the next two sections.
}

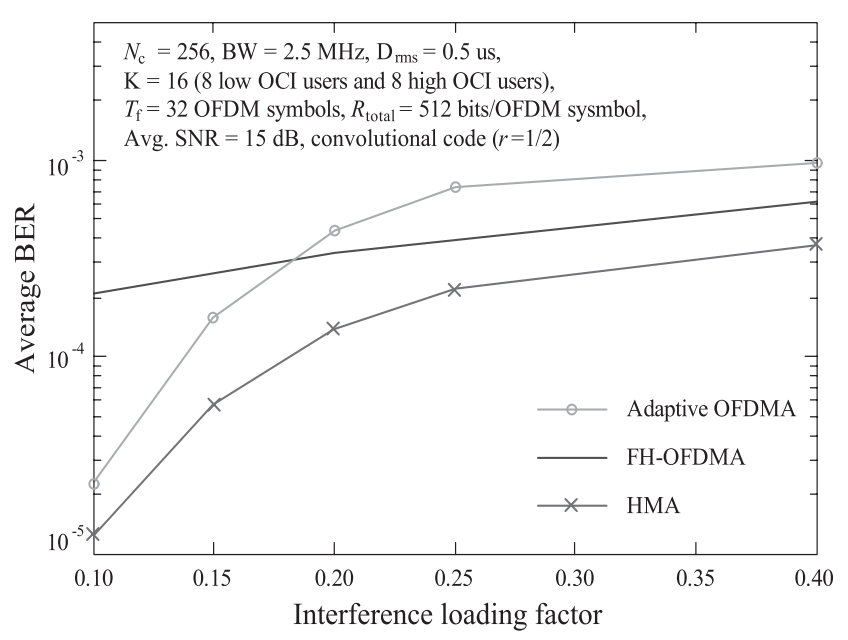

Fig. 2. Performance comparison of adaptive OFDMA, FH-OFDMA, and $\mathrm{HMA}$ in different $\mathrm{ICl}$ conditions.

in the neighboring cells. We took the same system configuration as for the Fig. 1 case and put eight users at the distance of $0.25 R$ from the cell center and eight users at the distance of $0.75 R$, for the cell radius $R .^{5}$ The figure plots the average values of BER for the eight users. From the figure, we observe that FH-OFDMA is better than adaptive OFDMA in the high OCI environment.

In the HMA framework, we assign multiple FH-OFDMA channels to the nomadic users that require high-data rate RT services in a high OCI environment, since the frequency diversity gain of FH-OFDMA is more efficient than the multi-user diversity gain of D-OFDMA. In order to determine the environment class, each nomadic user estimates the number of sub-carriers required for both cases - namely, $N_{L A}$ when it uses link adaptation through D-OFDMA channels and $N_{\mathrm{FH}}$ when it uses FHOFDMA channels. An FH-OFDMA channel is allocated to the user if

$$
N_{\mathrm{FH}} \leq \alpha_{n} N_{L A},
$$

for a compensating factor $\alpha_{n}$. Usually, the value $\alpha_{n}=1$ suffices but a bigger value may be used to reflect the resource required for control and feedback overheads of the D-OFDMA channel. If $\alpha_{n}>1$, it means that the extra $\left(\alpha_{n}-1\right) N_{L A}$ subcarriers may be regarded as such overheads. Considering the required service rate of the RT services discussed in the previous subsection, we may express the decision criterion of using the FH-OFDMA channel for RT services as follows

$$
N_{\mathrm{FH}} \leq \max \left\{\alpha_{n} N_{L A}, S C_{L A}\right\}
$$

In case the system allows for high frequency reuse factor (FRF), we may use D-OFDMA with high FRF for high data rate RT services in a high OCI environment.

A summary of the above discussions is listed in Table 1. The table, in effect, works as the definition of the proposed HMA

\footnotetext{
${ }^{5}$ We do not consider the users at the cell boundary (i.e., at $1 R$ distance), as those users are supposed to select the FH-OFDMA channels under the HMA framework.
} 
Table 1. Classification for hybrid multiple access scheme.

\begin{tabular}{|c|c|c|c|c|}
\hline \multicolumn{4}{|c|}{ Class } & \multirow{2}{*}{$\begin{array}{l}\text { Multiple } \\
\text { access }\end{array}$} \\
\hline Mobility & & vice & Environment & \\
\hline \multirow{4}{*}{$\begin{array}{c}\text { Nomadic } \\
\text { user }\end{array}$} & \multirow{3}{*}{$\begin{array}{l}\text { Real-time } \\
\text { service }\end{array}$} & \multirow{2}{*}{$\begin{array}{l}\text { High data } \\
\text { rate service }\end{array}$} & Low OCI & D-OFDMA \\
\hline & & & High OCI & FH-OFDMA* \\
\hline & & \multicolumn{2}{|c|}{ Low data rate service } & FH-OFDMA \\
\hline & \multicolumn{3}{|c|}{ Non real-time service } & S-ODFMA \\
\hline Mobile user & \multicolumn{3}{|c|}{ All types } & FH-ODFMA \\
\hline
\end{tabular}

* D-OFDMA with a high FRF value can be used optionally.

framework. Once a user condition is determined, in terms of mobility, service, and environment, we may look up in the table to find the preferred multiple access scheme.

\section{Other Considerations}

So far we have considered the issues fundamentally required for establishing the HMA framework. There are several other issues to consider in support of the HMA framework, namely, the issue of signaling and estimation errors, the issue of H-ARQ adaptation and the issue of applying MIMO technologies.

The error that occurs in the feedback channel towards the base station or in the channel estimation at mobile station can cause performance degradation in the HMA framework. Basically, each mobile is equipped with ARQ schemes that can compensate for the errors. However, if the performance degradation becomes large, we may adopt a readjustment process such that the OFDMA-channel (D-OFDMA or S-OFMDA channel) can be switched to some FH-OFDMA channels when the received throughput of the OFDMA channel drops below the expected throughput of the FH-OFDMA channels. Such a readjustment scheme can be used as a means to handle the problem of misestimating the mobility or the environment class of the mobile station and, in addition, the problem of large delay spread. ${ }^{6}$

The hybrid-ARQ (H-ARQ) mechanism [19] may provide a useful means to enhance the performance of the HMA scheme as it can help to compensate for mismatched link-adaptation, for the OFDMA and FH-OFDMA channels, or data frame errors by combining a channel coding and an ARQ scheme. The main effect of H-ARQ, however, is in elevating the received throughput towards the level that might be achieved if the fast-link adaptation is ideally applied. This indicates that the performance of an FH-OFDMA channel adopting H-ARQ may be hardly better than that of a D-OFDMA channel equipped with fast-link adaptation. Therefore, the HMA scheme can accommodate the $\mathrm{H}$ ARQ mechanism under the basic framework as defined above.

The MIMO technology [20] is an important physical layer tool that can help enhance the performance of the HMA scheme. We may adopt the MIMO technology in different forms in combination with the appropriate multiple access mechanisms considering the service requirements, mobility classes and environment classes. Detailed work on the usage of MIMO technology, as well as that of H-ARQ, is for future study.

\footnotetext{
${ }^{6}$ Note that a large delay spread can degrade the performance of OFDMA channels when the FG concept is applied. A more detailed discussion about the delay spread will be made in Section III.
}

\section{FRAME STRUCTURE DESIGN WITH FREQUENCY GROUPING}

The HMA framework as discussed above would possibly render an efficient and flexible multiple access mechanism for an OFDMA-based downlink system. However, the OFDMA-based system that utilizes multi-user diversity through link adaptation and scheduling inherently accompanies heavy control overhead. In this section, we discuss how to design the frame structure of the OFDMA-based downlink system to make it operate at reduced feedback and control overheads in both frequency and time domains. We focus the discussions on the D-OFDMA and S-OFDMA schemes, excluding the FH-OFDMA case that does not use fast link adaptation. As will be detailed below, we adopt the concept of frequency grouping as a means to reduce the overheads.

\section{A. Frequency Domain Design}

In broadband wireless OFDMA-based systems, the channel condition of each sub-carrier varies due to frequency selective fading. However, within the range of the coherence bandwidth, the fluctuation of the channel condition among the adjacent sub-carriers may be regarded as very small. So we divide the overall sub-carriers into frequency groups (FGs) of the same size, $S C_{\mathrm{FG}}$, by grouping the sub-carriers within each coherence bandwidth, and allocate one of the $S C_{\mathrm{FG}}$ sub-carriers in each FG as the pilot channel. Then we may regard that the SNR values are identical among all the sub-carriers belonging to the same FG and this SNR value can be determined by measuring the pilot channel. This enables channel feedback to be done per FG, instead of per sub-carrier. Consequently, the channel information of the $S C_{\mathrm{FG}}$ sub-carriers within an FG is represented by the pilot channel, so the amount of feedback information reduces to one per $S C_{\mathrm{FG}}$.

The coherence bandwidth, $B W_{C}$, is a function of the delay profile, which may be expressed as $B W_{C}=\frac{1}{\tau_{\max }}$ for the maximum delay spread $\tau_{\max }$. Table 2 lists the delay profiles, the related coherence bandwidth and the resulting number of subcarriers within the coherence bandwidth for the ITU, COST259, and $3 \mathrm{GPP}$ models. If we were to take all the models into consideration, the allowable FG size would be only 5 sub-carriers. However, this is too small a value for practical use and the reduction of feedback and pilot overheads is not large enough. Noting that, the objective of adopting the FG concept is to reduce the overhead of the D-OFDMA or the S-OFDMA channel used by nomadic users, we may exclude from consideration the models for mobile users such as vehicular model of ITU and the case 
Table 2. Standard delay profiles.

\begin{tabular}{|c|l|r|r|r|r|}
\hline Standard & Model & DRMS & Dmax & $\begin{array}{r}B W_{C} \\
(\mathrm{kHz})\end{array}$ & SCs* \\
\hline \multirow{5}{*}{ ITU } & $\begin{array}{l}\text { Indoor } \\
\text { office }\end{array}$ & $100 \mathrm{~ns}$ & $700 \mathrm{~ns}$ & 1429 & 146.3 \\
\cline { 2 - 6 } & Pedestrian & $750 \mathrm{~ns}$ & $3.7 \mu \mathrm{s}$ & 270 & 27.7 \\
\cline { 2 - 6 } Vehicular & $4 \mu \mathrm{s}$ & $20 \mu \mathrm{s}$ & 50 & 5.1 \\
\hline \multirow{5}{*}{$\begin{array}{c}\text { COST } \\
259\end{array}$} & $\begin{array}{l}\text { Typical } \\
\text { urban }\end{array}$ & $0.5 \mu \mathrm{s}$ & $2.14 \mu \mathrm{s}$ & 467 & 47.9 \\
\cline { 2 - 6 } & $\begin{array}{l}\text { Rural } \\
\text { area }\end{array}$ & $0.1 \mu \mathrm{s}$ & $528 \mathrm{~ns}$ & 1893 & 194.0 \\
\cline { 2 - 6 } & $\begin{array}{l}\text { Hilly } \\
\text { terrain }\end{array}$ & $3 \mu \mathrm{s}$ & $18.02 \mu \mathrm{s}$ & 56 & 5.7 \\
\hline \multirow{5}{*}{$3 \mathrm{GPP}$} & $\begin{array}{l}\text { Case } 1 \\
(3 \mathrm{~km} / \mathrm{h})\end{array}$ & $280 \mathrm{~ns}$ & $976 \mathrm{~ns}$ & 1025 & 105.0 \\
\cline { 2 - 6 } & $\begin{array}{l}\text { Case } \\
(3 \mathrm{~km} / \mathrm{h})\end{array}$ & $9.2 \mu \mathrm{s}$ & $20 \mu \mathrm{s}$ & 50 & 5.1 \\
\cline { 2 - 6 } & $\begin{array}{l}\text { Case 3 } \\
(120 \mathrm{~km} / \mathrm{h})\end{array}$ & $242 \mathrm{~ns}$ & $781 \mathrm{~ns}$ & 1280 & 131.0 \\
\cline { 2 - 6 } & $\begin{array}{l}\text { Case 4 } \\
(3 \mathrm{~km} / \mathrm{h})\end{array}$ & $488 \mathrm{~ns}$ & $976 \mathrm{~ns}$ & 1025 & 105.0 \\
\cline { 2 - 6 } & $\begin{array}{l}\text { Case 5 } \\
(50 \mathrm{~km} / \mathrm{h})\end{array}$ & $280 \mathrm{~ns}$ & $976 \mathrm{~ns}$ & 1025 & 105.0 \\
\cline { 2 - 6 } & $\begin{array}{l}\text { Case 6 } \\
(250 \mathrm{~km} / \mathrm{h})\end{array}$ & $240 \mathrm{~ns}$ & $781 \mathrm{~ns}$ & 1280 & 131.1 \\
\cline { 2 - 6 } & $\begin{array}{l}\text { Case 7 } \\
(50 \mathrm{~km} / \mathrm{h})\end{array}$ & $4 \mu \mathrm{s}$ & $14.48 \mu \mathrm{s}$ & 69 & 7.0 \\
\hline
\end{tabular}

* The number of subcarriers in $B W_{C}$ when 2,048 subcarriers lie in $20 \mathrm{MHz}$ band.

3,5,6, and 7 models of 3GPP. In addition, we may also exclude the hilly terrain model of COST259 by assuming that the users under this model can be serviced by the FH-OFDMA channel. This assumption is reasonable as the readjustment mechanism in the HMA framework described in Section II-D is supposed to switch an OFDMA channel with large delay spread to some FH-OFDMA channels when the received throughput of the OFDMA channel is lower than the expected throughput of the FH-OFDMA channels. Therefore, it is adequate in the FG design to take into account the RMS delay spread below $1 \mu \mathrm{s}$, expecting a performance degradation below $1.5 \mathrm{~dB}$. In case a user suffers from a delay spread larger than $1 \mu \mathrm{s}$, the readjustment mechanism of the HMA framework will change the multiple access scheme to FH-OFDMA.

Fig. 3 shows the simulated performance of the adaptive OFDMA for nomadic users with various different delay spreads and FG sizes. For the simulations we assumed an OFDMA system with 2,048 sub-carriers in a $20 \mathrm{MHz}$ band at a $2 \mathrm{GHz}$ frequency and a $115.2 \mu$ s symbol length including a $12.8 \mu$ s cyclic prefix (CP). We used a 5-path Rayleigh fading channel with exponential decaying delay profile and used five RMS delay spread values of $0.5,1,2,3$, and $4 \mu \mathrm{s}$. We set the path loss exponent to 3.5 and assumed perfect channel estimation and no feedback error. The figure plots the SNR values required to achieve the data rate of 8,192 bits/OFDM symbol with a $10^{-5}$ BER constraint. We used QPSK, 16-QAM, and 64-QAM for modulation schemes and a rate $1 / 2$ convolutional code with length 7

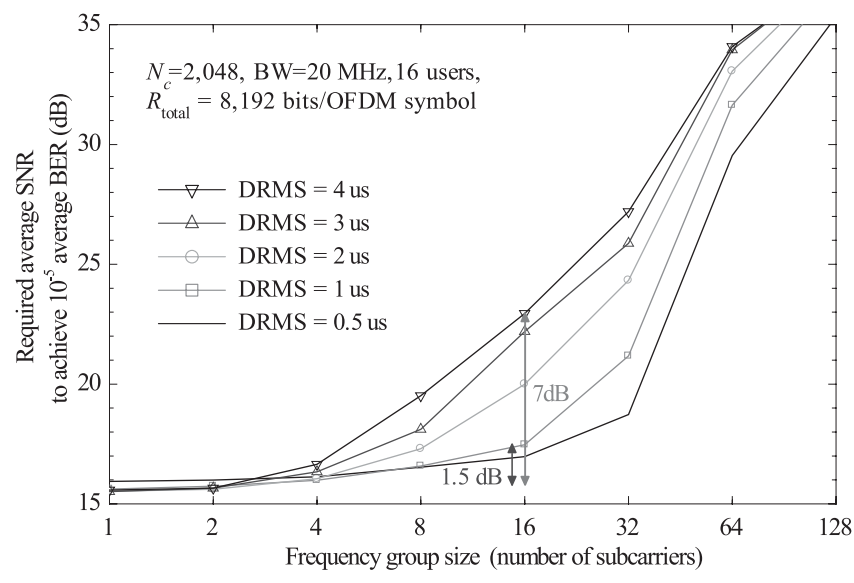

Fig. 3. Performance of adaptive OFDMA for various delay spread and FG size values.

for channel coding. We assumed equal power allocation and fixed code rate and applied the ASA and ABA algorithms [17] to adapt the modulation set. Plotted in the figure are the average values of 16 users.

Based on the SNR performances displayed in the figure, we take the prime number 17 as the value of $S C_{\mathrm{FG}}$, as the performance degradation falls within $1.5 \mathrm{~dB}$ range for $1 \mu \mathrm{s}$ RMS delay spread and within $7 \mathrm{~dB}$ range for the worst case of $4 \mu$ sMS delay spread for this value. Then the feedback overhead reduces to 1/17 through the FG based feedback operation, while the multiuser diversity gain obtained from the adaptive OFDMA gets degraded by $1.5 \mathrm{~dB}$. The choice of the prime number 17 is intended to include a pilot channel and a radix-2 number of data channels and, in addition, for an easy adoption of the intra frequency group hopping (IFGH) technique to be discussed in the next section. ${ }^{7}$

As a result, we get the basic framework of the HMA based downlink system as follows: Given a $20 \mathrm{MHz}$ band at a $2 \mathrm{GHz}$ frequency, we put 2,048 sub-carriers in it. The symbol duration is then determined by the inverse of the sub-carrier spacing to be $102.4 \mu \mathrm{s}(=2,048 / 20 \mathrm{MHz})$. If we add a $1 / 8 \mathrm{CP}$ overhead of $12.8 \mu \mathrm{s}$, the resulting symbol length becomes $115.2 \mu \mathrm{s}$. We divide the 2,048 sub-carriers into 120 FGs of 17 sub-carriers each (i.e., 2,040 sub-carriers in total) and 8 null sub-carriers, ${ }^{8}$ with each FG composed of 1 pilot channel and 16 data channels. Under this HMA framework, we build a D-OFDMA channel with at least $S C_{L A}$ sub-carriers within the same FG and an S-OFDMA channel using an FG. For the FH-OFDMA channels, we build an FH-OFDMA channel group with $S C_{\mathrm{FH}}$ sub-carriers taken from $S C_{\mathrm{FH}}$ different FGs. ${ }^{9}$ One FH-OFDMA channel group can accommodate $S C_{\mathrm{FH}}$ FH-OFDMA channels. It is expected in general that different multiple access schemes use dif-

\footnotetext{
${ }^{7}$ For the design of an efficient frequency hopping pattern, the prime-numbered hopping period is preferred.

${ }^{8}$ These null sub-carriers may be used for carrying fixed pilot signals or for other purpose. We do not specify guard bands here, but some FGs may be arranged as guard bands as necessary.

${ }^{9}$ It is necessary to maintain some distance between two adjacent FGs among the $S C_{\mathrm{FH}} \mathrm{FGs}$ in order to provide frequency diversity. We set the minimum distance to $10 \mathrm{FGs}$ in the HMA-based downlink system.
} 


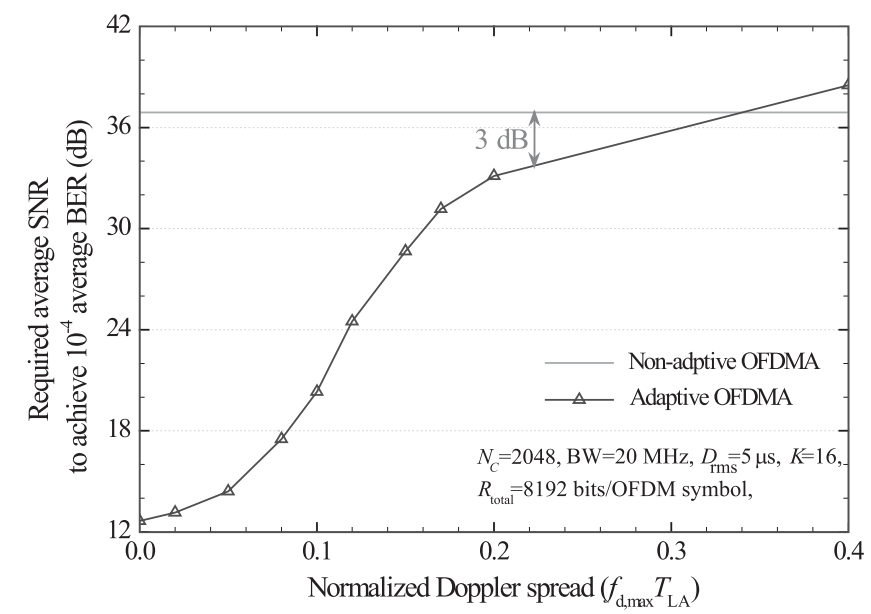

Fig. 4. Performance comparison of Adaptive and Non-adaptive OFDMAs with respect to normalized Doppler spread.

ferent FGs in the HMA framework, but it is not restricted. ${ }^{10}$

The adoption of FG concept may reduce the frequency and interference diversity as it reduces the number of independent sub-channel. However, after applying the FG concept, there still exist 120 FGs (case of 2,048 sub-carriers) that can be expected to provide sufficient frequency and interference diversity.

\section{B. Time Domain Design}

In the time domain, we take the frame as the unit of link adaptation. However, the coherence time required for link adaptation, $T_{L A}$, should consider the feedback delay since the link adaptation is performed based on the feedback information reported by the user. Therefore, $T_{L A}$ is determined by

$$
T_{L A}=T_{f}+T_{f b},
$$

where $T_{f}$ and $T_{f b}$ denote the frame duration and the feedback delay, respectively. In the HMA-based downlink system design, we assumed that the maximum feedback delay is $T_{f}$, and set $T_{L A}$ to $2 T_{f}$. As mentioned above, the $T_{L A}$ value should be determined such that the nomadic users can apply link adaptation techniques. The target nomadic users are those moving at a speed below $30 \mathrm{~km} / \mathrm{h}$. Fig. 4 shows the simulated performance of the adaptive OFDMA according to the normalized Doppler spread defined by

$$
f_{d, \text { norm }}=f_{d, \max } \cdot T_{L A},
$$

where $f_{d \text {,max }}$ denotes the maximum Doppler spread. It plots the SNR values required to achieve the data rate of 8,192 bits/OFDM symbol with a $10^{-5}$ BER constraint and a $4 \mu$ s RMS delay spread. For the other parameters, we took the same system configuration as for the Fig. 3 case. Plotted in the figure are the average values of 16 users. Based on the SNR performances exhibited in the figure, we take 0.23 as the normalized Doppler spread value, for which the performance of the adaptive OFDMA has a gain of about $3 \mathrm{~dB}$ when compared with the nonadaptive OFDMA case. As the target maximum Doppler spread

\footnotetext{
${ }^{10}$ For example, D-OFDMA channels may use the remaining sub-carriers in
} the FH-OFDMA FGs.

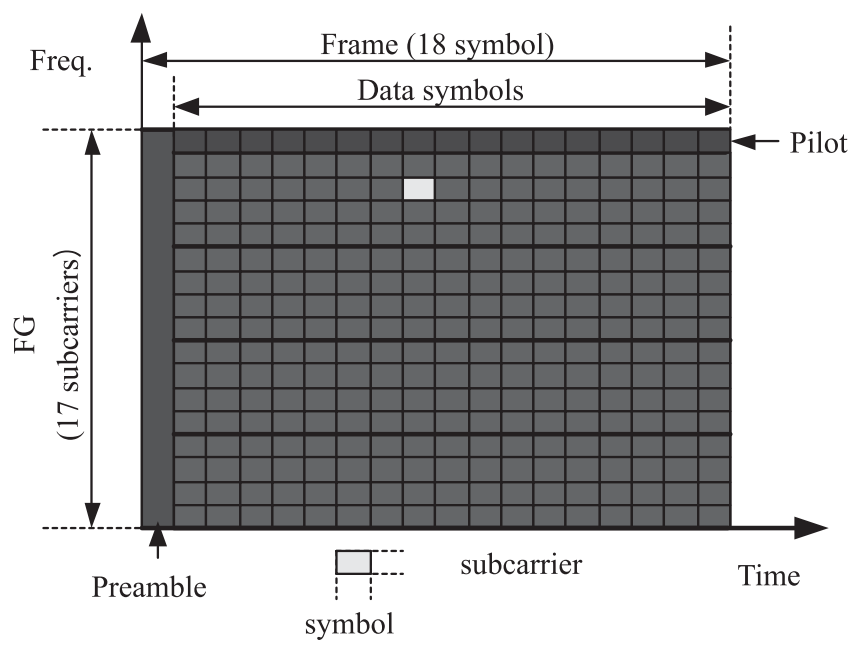

Fig. 5. Downlink frame structure.

is $55.55 \mathrm{~Hz}$ at the $30 \mathrm{~km} / \mathrm{h}$ speed, the corresponding $T_{L A}$ value is determined to be $4.147 \mathrm{~ms}$ (or 36 OFDM symbols) and the $T_{f}$ value to be 18 OFDM symbols.

We take the frame structure to be composed of 1 preamble symbol and 17 data symbols. The preamble symbol is for use in estimating the frequency offset and performing the time synchronization. The symbol number 17 is taken to match with the FG size of 17 sub-carriers as the IFGH technique uses an $n$ by $n$ hopping matrix for a prime number $n .{ }^{11}$ From the resulting frame length, we can calculate the channel coherence time of user $i, C C T_{i}$, to be $0.23 / f_{d, \max }$. A user $i$ is regarded as nomadic if $C C T_{i} \leq T_{L A}(=4.147 \mathrm{~ms})$ in the HMA framework.

Fig. 5 depicts the frame structure of the HMA framework in frequency and time domains, which is defined in the form of an FG consisting of 17 sub-carriers in frequency domain and in the form of a frame consisting of 18 symbols in time domain. In this design, the control overhead per frame is 1 preamble symbol in each of the sub-carriers and 1 sub-carrier in each of the 17 data symbols for the pilot channel. Therefore, the total control overhead is calculated to be $1 / 18+1 / 17 \times(17 / 18)$, which amounts to $11.1 \%$.

\section{INTRA-FG AVERAGING FOR MULTI-CELL ENVIRONMENT}

The frequency and time domain structure discussed above was designed to reduce the feedback and control overheads of the adaptive OFDMA system. However, in the multi-cell OFDMA environment, the channel quality also depends on how the neighboring cells use their sub-carriers. Consequently, even the sub-carriers within the same FG may experience different SINR due to the resulting OCI difference. In this circumstance, the pilot of an FG fails to represent the correct channel quality of the sub-carriers within the FG. In order to overcome such OCI variation and to effectively utilize the FG concept in multicell OFDMA systems, we employ intra frequency group averaging (IFGA) techniques, namely, intra frequency group hop-

\footnotetext{
${ }^{11}$ The details of the IFGH technique will be discussed in the next section.
} 


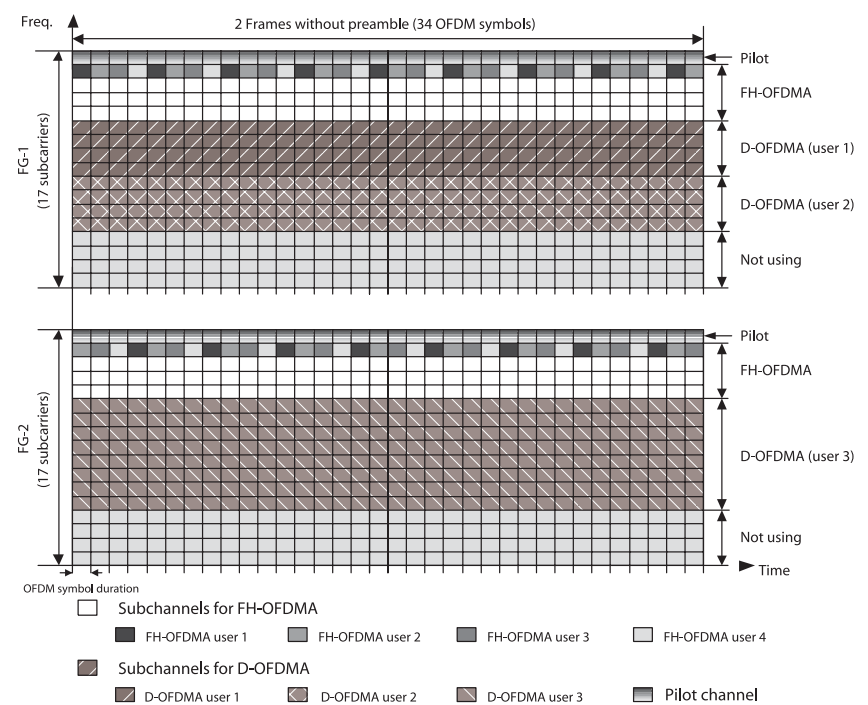

(a)

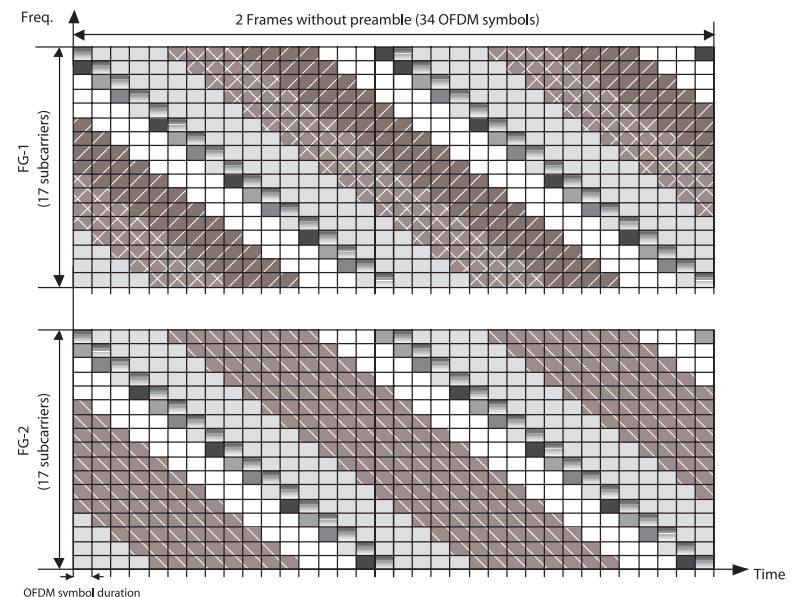

(b)

Fig. 6. Illustration of IFGH; (a)original spectrum of two FGs, (b)spectrum after frequency-hopping.

ping (IFGH) and intra frequency group spreading (IFGS). These IFGA techniques are intended to average out the sub-channels in an FG by adopting frequency hopping (in the case of IFGH) or frequency-domain spreading (in the case of IFHS) mechanisms. Then the OCI caused by the allocation of sub-channels at the neighboring cells gets spread over all the sub-channels within the FG, and, consequently, the channel quality of the IFGAapplied sub-channels within an FG becomes uniform, with the IFGA-applied pilot duly representing the actual SINR of the constituent sub-channels.

\section{A. Intra-FG Hopping}

IFGH technique uses frequency hopping to average out the sub-channels. It spreads only the 17 by 17 data symbol array by using the 17 by 17 cell-specific Latin sequence. Once IFGH is applied, each sub-channel of a cell is distinguished by the hopping index and the signal from the other cells is distinguished by the cell-specific hopping sequence.

For channel estimation, we can process the pilot signal as it is hopped by the same sequence that is used for the data channels. While such channel estimation is adequate enough for resource management algorithms, it is not good enough for the OFDM systems since the OFDM receiver requires compensating the channel response of each sub-carrier through channel equalization. In order to estimate and compensate the channel response of the $(i, j)$-th OFDM element, which represents the $j$-th tone (i.e., sub-carrier) at the $i$ th OFDM symbol, we adopt the 2-dimensional minimum mean square error (2-D MMSE) estimation method [18]. In the 2-D MMSE equalizer, the channel response of each OFDM element is estimated through the pilot elements within the coherence bandwidth and the coherence time ranges of the desired element. The receiver applies the interpolation and filtering methods using the auto-correlation functions between the pilot elements and the cross-correlation functions between the pilot element and the desired element.

Fig. 6 illustrates the IFGH technique; (a) shows the original sub-channel structure of two FGs and two frames and (b) shows the sub-channel structure after applying cell specific hopping. The preamble symbol is omitted in the figure as the IFGH technique is applied only to the data symbols. As shown in Fig. 6(b), the used and unused channels are well spread out to all the subcarriers within each FG. Therefore, both the SNR and SINR responses are well averaged out, since IFGH is applied to the signals from other cells as well as to the signal from the desired cell.

\section{B. Intra-FG Spreading}

IFGS technique uses frequency-domain spreading to average out the sub-channels. It uses Walsh-Hadamard sequence to distinguish the data sub-channels of a cell and applies a cellspecific scrambling code to distinguish the cells.

In contrast to the IFGH that spreads the pilot channel with the same hopping sequence, IFGS leaves out the pilot channel from spreading. It only spreads the 16 data channels. The pilot channel is spread by frequency hopping as in the IFGH case and then inserted to the frequency-domain spread data channels. Fig. 7 illustrates this procedure. Fig. 7(a) shows the frequencydomain spread 16 data sub-channels (excluding the pilot channel) for the original sub-channel structure in Fig. 6(a). In order to demonstrate the pilot insertion procedure, we redraw Fig. 7(a) to a simplified figure Fig. 7(b), where each block represents the code-multiplexed signal of 16 code domain signals in Fig. 7(a). Fig. 7(c) depicts the final form of the IFGS spectrum, including the frequency-hopped pilot.

Note that in the last figure the frequency-hopped pilot is inserted among the code-spread data channels. The reason of using the frequency-hopped pilot is because it is hard to compensate channel response at the OFDM receiver if a frequencydomain spread pilot is used. When the pilot elements are spread in the frequency domain, it is possible to measure the channel response after despreading but, for this despreading process, we need to compensate channel response to remove the inter-code interference.

\section{Performance Comparison}

We conducted computer simulations to compare the performances of IFGH and IFGS. For the simulations we took the 7- 


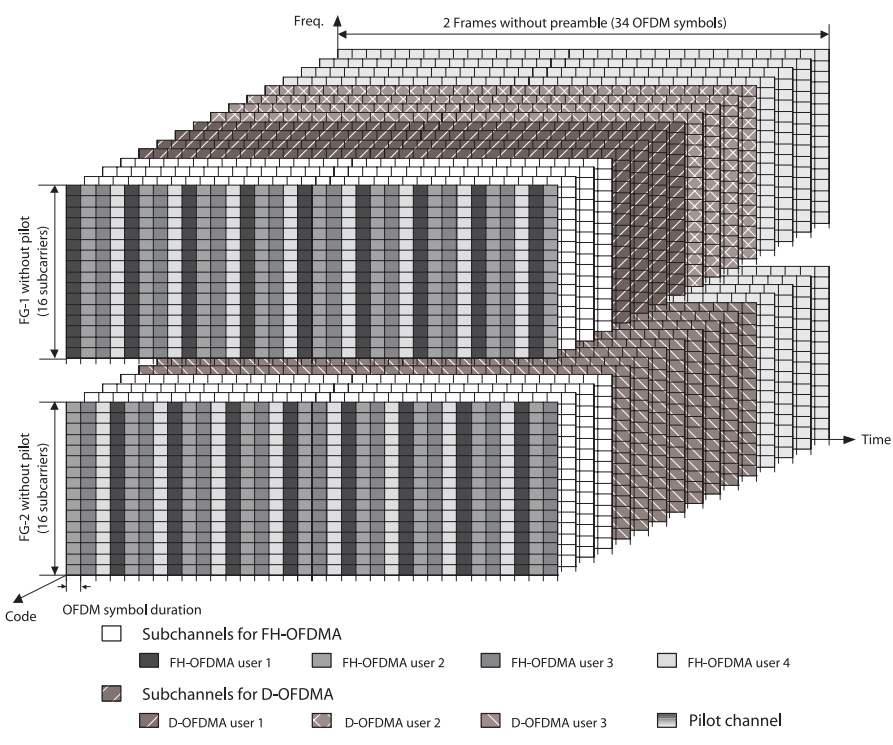

(a)

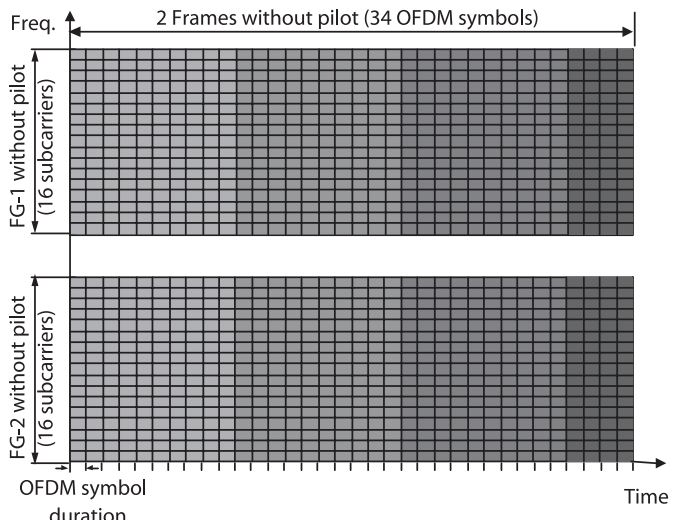

(b)

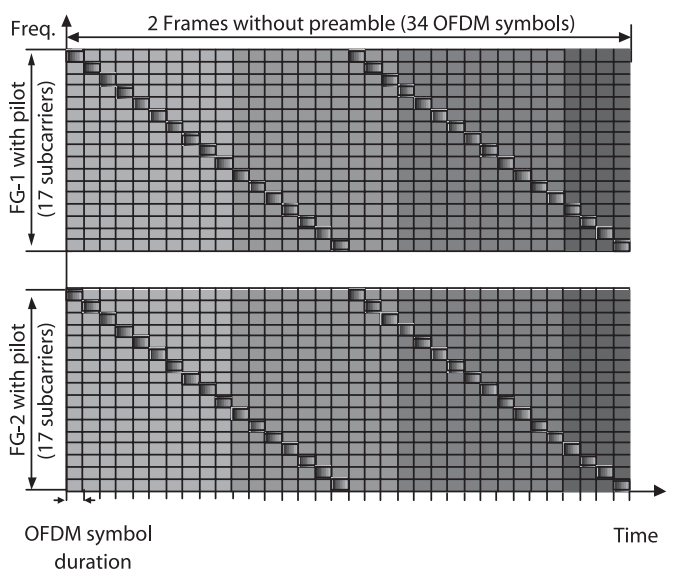

(c)

Fig. 7. Illustration of IFGS; (a) frequency-domain spread data subchannels, (b) simplified figure of (a) before pilot insertion, (c) IFGS spectrum after frequency-hopped pilot insertion.

cell environment composed of the target cell and its six first-tier neighbors. We assumed that each cell uses 2,048 sub-carriers in a $20 \mathrm{MHz}$ band at a $2 \mathrm{GHz}$ frequency and the symbol length is
115.2 $\mu$ s including a 12.8 $\mu$ s CP. We used a 5-path Rayleigh fading channel with an exponential power delay profile and set the RMS delay spread and the path loss exponent to $1 \mu \mathrm{s}$ and 3.5, respectively. We assumed that the maximum Doppler spread is $55.55 \mathrm{~Hz}$, which represents a $30 \mathrm{~km} / \mathrm{h}$ mobile speed. This setting corresponds to the worst case considered in the frequency and time domain design of Section III. We compared the performances of six different schemes: 1) IFGH with perfect channel state information (CSI), 2) IFGH with 2D-MMSE channel estimation, 3) IFGS with perfect CSI, 4) IFGS with 2DMMSE channel estimation, 5) an ideal adaptive OFDMA, and 6) an FG-based adaptive OFDMA without IFGA techniques. For the ideal OFDMA scheme, we assumed that the base station knows all the channel information of each sub-carrier and applies link adaptation for each sub-carrier. For the FG-based OFDMA scheme, we assumed that the base station knows the averaged channel information of an FG and applies link adaptation FG based but does not use the IFGA technique. For the IFGS and IFGH schemes, we applied the same assumption as for the FG-based adaptive OFDMA case except that the base station uses IFGA technique. We also assumed that the FG-based adaptive OFDMA scheme uses a 2D-MMSE equalizer and the IFGS schemes use a frequency-hopped pilot.

Fig. 8 shows the resulting averaged BER performances when link adaptation is performed among the modulation set QQPSK, 16-QAM, and 64-QAM $\}$ to achieve the data rate of 8,192 bits/OFDM symbol using a rate $1 / 2$ convolutional code with length 7 as the channel coding scheme. For the link adaptation, we assumed equal power allocation and fixed code rate and used the ASA and ABA algorithms [17]. We used the OCI model of Fig. 2 and applied the IFGS and IFGH techniques to the neighboring cells only when the corresponding technique is applied in the desired cell. Fig. 8(a) shows the BER performances at the locations of $0.25 R$ and $0.5 R$ from the cell center with the ILF value set to 0.4; and Fig. 8(b) shows the BER performances at the location of $0.5 R$ from the cell center for the ILF values of 0.125 and 0.875 . As the IFGS and IFGH techniques are designed for D-OFDMA and S-OFDMA users, the performance of the users at the cell boundary region (i.e., $0.5 R \sim 1 R$ ), which would select the FH-OFDMA channels is not considered.

From the figures, we observe that the performance of IFGH scheme is comparable to that of the IFGS scheme in general but becomes superior when channel condition becomes good. We also observe that the BER performance of IFGH approaches the ideal case. The performance gap between the two is inherently unavoidable as the concept of frequency grouping is adopted to reduce the feedback and pilot overheads at the sacrifice, to some extent, of the performance of the adaptive OFDMA. From the two figures we can find that the performance further degrades to the level of practical adaptive OFDMA if frequency grouping is applied alone, without the IFGA technique. These results demonstrate that the IFGA technique is an enabler of the FG concept that makes FG successfully applicable even in the multi-cell environment. 


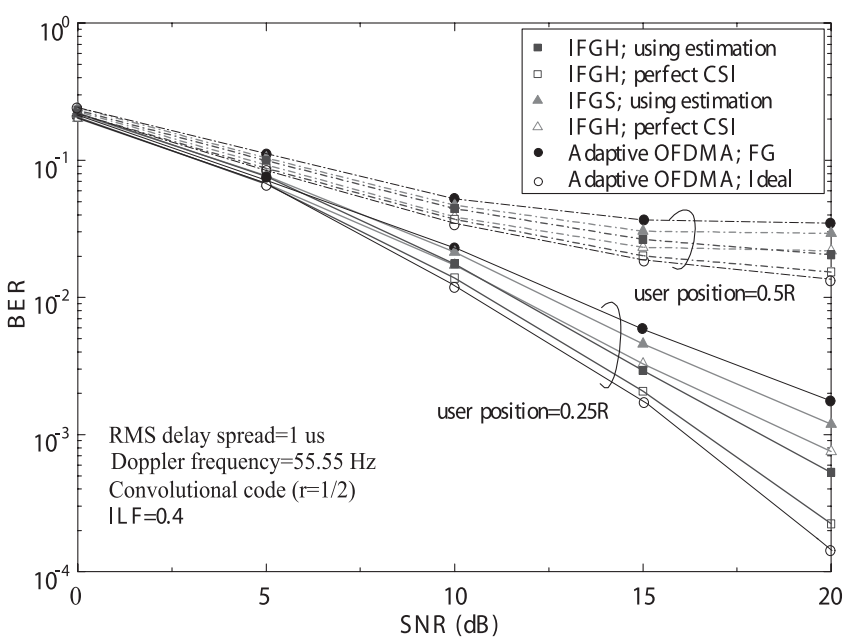

(a)

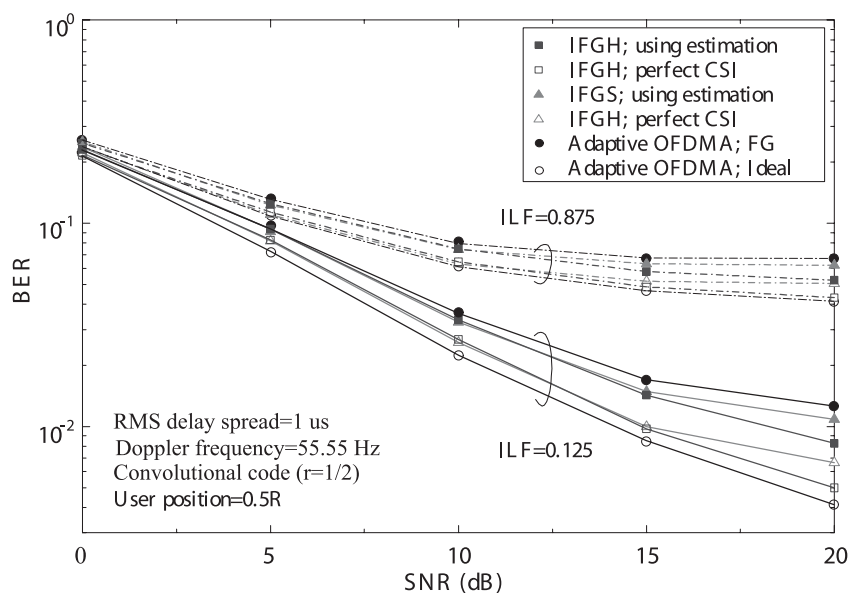

(b)

Fig. 8. Performance comparison of IFGH and IFGS; (a) for different user positions, (b) for different ILF values.

\section{CONCLUSION}

In this paper, we have discussed how to design an efficient and flexible next-generation wireless communication system on the basis of OFDMA. Focusing on the downlink system design, we detailed the reasoning for adopting the HMA framework and the frame structure in connection with the concept of FG and IFGA techniques. The resulting downlink system is therefore tailored for accommodating various user requirements in diverse environments, with flexible adaptation of multiple access schemes and resource management strategies. It also renders an efficient structure that operates at reduced feedback and control overheads and is robust to the OCI in the multi-cell environment.

In the proposed downlink system, the HMA framework plays a central role for building an efficient and flexible downlink system. It basically combines various different multiple access mechanisms as the basis for an OFDMA system and chooses the most appropriate multiple access mechanism depending on the mobility, services, and environment of the user. Conducting a rigorous reasoning process, we have generated the criteria for selecting the best multiple access technique for each given situation and summarized it in Table 1 . Therefore, by employing the HMA framework, we can effectively accommodate sophisticated user requirements in diverse user environments.

The conception of FG and its extension to IFGA processing were intended to reduce the huge feedback and control overheads that are normally involved in designing an adaptive OFDMA system. By forming an FG by combining the subcarriers within a coherence bandwidth, we could regard that the channel condition becomes identical among all sub-carriers within the same FG; and by arranging an IFGA processing to average out the channel characteristics of each constituent subchannel within the same FG, we could overcome the OCI variation problem caused by the non-uniform resource usage of the neighboring cells in the multi-cell environment. According to computer simulations, it turned out that the FG concept can truly achieve the goal of reducing the feedback and control overheads without sacrificing the system performance, in the multi-cell environment, when IFGA technique is applied in conjunction with FG.

The proposed OFDMA downlink system design may put a corner stone in investigating how to flexibly and efficiently accommodate the various user requirements, mobility, and environments in one framework, while reducing the control and feedback overheads of link adaptation techniques and making the system robust to OCI. However, it deals mainly with the system-level downlink data channel design, so should be complemented by an uplink and control channel design. In addition, a lower-level design should follow shich should include the application of the H-ARQ and MIMO technologies.

\section{ACKNOWLEDGEMENT}

We gratefully acknowledge the valuable comments and discussions of the SNU 4G Research Team lead by Professors Kwang Bok Lee and Saewoong Bahk. In particular, we deeply appreciate the assistance of Mr. Jungmin Choi in designing the IFGA scheme and conducting the simulations.

\section{REFERENCES}

[1] ITU, "Report of eighth meeting of ITU-R working party 8F," Ottawa, Japan, May-June 2002.

[2] R. Nee and R. Prasad, OFDM for Wireless Multimedia Communications, Artech House, 2000

[3] H. Rohling and R. Funeid, "Performance comparison of different multiple access schemes for the downlink of an OFDM communications system," in Proc. IEEE VTC'97, 1997.

[4] H. Sari, "Orthogonal frequency division multiple access with frequency hopping and diversity," in Proc. Inter. Workshop Multi-Carrier SpreadSpectrum (MC-SS), 1997.

[5] R. Laroia, J. Li, and M. D. Vanderveen, "Orthogonal frequency division multiplexing based spread spectrium multiple access," US Patents no. 6,473,418 B1, Oct. 2002

[6] S. Hara and R. Prasad, "Design and performance of multicarrier CDMA system in frequency-selective Rayleigh fading channels," IEEE Trans. Veh. Technol., vol. 48, no. 5, 1999.

[7] H. Atarashi, S. Abeta, and M. Sawahashi, "Variable spreading factororthogonal frequency and code division multiplexing (VSF-OFCDM) for broadband packet wireless access," IEICE Trans. Commun., vol. E86-B, no. 1,2003

[8] S. Kaiser, "MC-FDMA and MC-TDMA versus MC-CDMA and SSMC-MA: Performance evaluation for fading channels," in Proc. IEEE ISSSTA'98, 1998.

[9] A. Jalali, R. Padovani, and R. Pankaj, "Data throughput of CDMA-HDR a high efficiency-high data rate personal communication wireless system," in Proc. IEEE VTC 2000, 2000. 
[10] X. Liu, E. K. P. Chong, and N. B. Shroff, "Opportunistic transmission scheduling with resource-sharing constraints in wireless networks," IEEE J. Select. Areas Commun., vol. 19, no. 10, 2001.

[11] S. Borst and P. Whiting, "Dynamic rate control algorithms for HDR throughput optimization," in Proc. IEEE INFOCOM 2001, 2001.

[12] D. Park et al., "A new wireless packet scheduling algorithm based on the CDF of user transmission rates," in Proc. IEEE GLOBECOM 2003, 2003.

[13] X. Liu, E. K. P. Chong, and N. B. Shroff, "A framework for opportunistic scheduling in wireless networks," Computer Networks, vol. 41, 2003.

[14] C. Y. Wong et al., "OFDMA with adaptive subcarrier, bit, and power allocation," IEEE J. Select. Areas Commun., vol. 17, no. 10, 1999.

[15] W. Rhee and J. M. Cioffi, "Increase in capacity of OFDMA system using dynamic subchannel allocation," in Proc. IEEE VTC 2000 Spring, 2000.

[16] D. Kivanc, G. Li, and H. Liu, "Computationally efficient bandwidth allocation and power control for OFDMA," IEEE Trans. Wireless Commun., vol. 2, no. 6, 2003.

[17] H. S. Kim et al., "Efficient subcarrier and bit allocation algorithm for OFDMA system with adaptive modulation," in Proc. IEEE VTC 2004 Spring, 2004.

[18] O. Edfors et al., "OFDM channel estimation by singular value decomposition," in Proc. IEEE VTC'96 Spring, 1996.

[19] S. Kallel and D. Haccoun, "Generalized type II hybrid ARQ scheme using punctured convolutional coding," IEEE Trans. Commun., vol. 38, no. 11, 1990.

[20] A. J. Paulraj et al., "An Overview of MIMO Communications - a key to gigabit wireless," Proc. IEEE, vol. 92, no. 2, 2004.

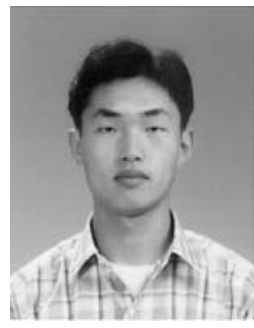

Won-Ick Lee received the B.S. and M.E. degrees in electrical engineering from Seoul National University, Seoul, Korea, in 1998 and 2000, respectively. He spent the summer of 1998 as an intern at ETRI(Electronics and Telecommunications Research Institute). He is currently working toward the Ph.D. degree in electrical engineering and computer science at Seoul National University. His current research interests include QoS, routing algorithm, wireless MAC protocol, wireless sensor networks, and resource allocation in wireless/wired networks.

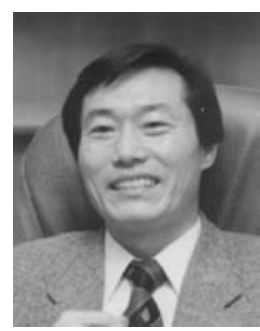

Byeong Gi Lee received the B.S. and M.E. degrees from Seoul National University, Seoul, Korea, and Kyungpook National University, Daegu, Korea, both in electronics engineering, and the Ph.D. degree in electrical engineering from the University of California, Los Angeles. He was with Electronics Engineering Department of ROK Naval Academy as an Instructor and Naval Officer in active service from 1974 to 1979 , and worked for Granger Associates, Santa Clara, CA, as a Senior Engineer responsible for applications of digital signal processing to digital transmission from 1982 to 1984 , and for AT\&T Bell Laboratories, North Andover, MA as a Member of Technical Staff responsible for optical transmission system development along with related the standards works from 1984 to 1986 . He joined the faculty of Seoul National University in 1986 and served as the Director of the Institute of New Media and Communications in 2000 and the Vice Chancellor for Research Affairs from 2000 to 2002.

Dr. Lee was the founding chair of the Joint Conference of Communications and Information (JCCI), the chair of the Steering Committee of the Asia Pacific Conference on Communications (APCC), and the chair of the founding committee of the Accreditation Board for Engineering Education of Korea (ABEEK). He served as the President of Korea Society of Engineering Education (KSEE) and as a Vice President of Korea Institute of Communication Sciences (KICS). He was the editor of the IEEE Global Communications Newsletter, an associate editor of the IEEE Transactions on Circuits and Systems for Video Technology, and the founding Associate Editor-in-Chief and the EIC of the Journal of Communications and Networks (JCN). He served for the IEEE Communications Society as the Director of Membership Programs Development, as the Director of Asia Pacific Region, as the Director of Magazines and as a Member-at-Large to the Board of Governors. He currently serves a Vice President of the ABEEK and as the TPC Chair of IEEE International Conference on Communications (ICC) 2005 .
Dr. Lee is a co-author of Broadband Telecommunication Technology, 1st \& 2nd editions, (Artech House, 1993 \& 1996), Scrambling Techniques for Digital Transmission (New York: Springer Verlag, 1994), Scrambling Techniques for CDMA Communications (Norwell, MA: Kluwer Publisher, 2001), and Integrated Broadband Networks (Norwood, MA: Artech House, April 2002). He holds seven U.S. patents with four more patents pending. His current fields of interest include broadband networks, wireless networks, communication systems, and signal processing.

He received the 1984 Myril B. Reed Best Paper Award from the Midwest Symposium on Circuits and Systems, Exceptional Contribution Awards from AT\&T Bell Laboratories, a Distinguished Achievement Award from Korea Institute of Communication Sciences (KICS), and the 2001 National Academy of Science (of Korea) Award. He is a Member of the National Academy of Engineering of Korea, a Member of Sigma Xi, and a Fellow of the IEEE.

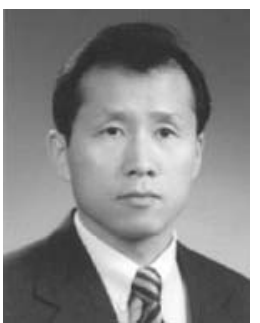

Kwang Bok Lee received the B.A.Sc. and M.Eng. degrees from the University of Toronto, Toronto, ON, Canada, in 1982 and 1986, respectively, and the $\mathrm{Ph} . \mathrm{D}$. degree from McMaster University, Hanilton, ON, Canada in 1990. He was with Motorola Canada from 1982 to 1985, and Motorola USA from 1990 to 1996, as a Senior Staff Engineer. At Motorola, he was involved in the research and development of wireless communication systems. He was with Bell-Northern Research, Canada, from 1989 to 1990. In March 1996 he joined the School of Electrical Engineering, Seoul National University, Seoul, Korea, where he is currently an Associate Professor. He was a Vice Chair of the School of Electrical Engineering from 2000 to 2002. He has been serving as a Consultant to a number of wireless industries. His research interests include mobile communications, communication theories, spread spectrum, and signal processing. He holds ten U.S. patents and two Korean patents, and has a number of patents pending.

Dr. Lee was an Editor of the IEEE Journal of Selected Areas in Communications, Wireless Series, in 2001, and is currently an Editor of the IEEE Transactions on Wireless Communications and the Journal of Communications and Networks. He received the Best Paper Award at the CDMA International Conference in 2000 .

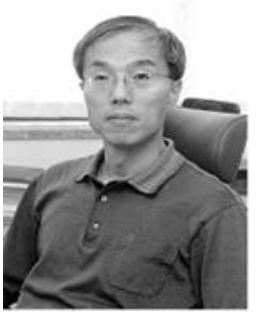

Saewoong Bahk received the B.S. and M.S. degrees in electrical engineering from Seoul National University, Seoul, Korea, in 1984 and 1986, respectively, and the Ph.D. degree in systems engineering from the University of Pennsylvania, Philadelphia, in 1991. He worked in the field of Network Management at AT\&T Bell Laboratories as a Member of the Technical Staff from 1991 through 1994. Since 1994, he has been with the School of Electrical Engineering at Seoul National University and currently serves as an Associate Professor. He is an Editor of Journal of Communications and Networks. His areas of interests include performance analysis of communication networks, network protocol design, and resource management at wireless/wired/WDM networks. 